University of Texas at El Paso

ScholarWorks@UTEP

\title{
Towards Improved Trapezoidal Approximation to Intersection (Fusion) of Trapezoidal Fuzzy Numbers: Specific Procedure and General Non-Associativity Theorem
}

\author{
Gang Xiang \\ Vladik Kreinovich \\ The University of Texas at El Paso, vladik@utep.edu
}

Follow this and additional works at: https://scholarworks.utep.edu/cs_techrep

Part of the Computer Engineering Commons

Comments:

Technical Report: UTEP-CS-10-02a

Published in the Proceedings of the IEEE World Congress on Computational Intelligence WCCI'2010, Barcelona, Spain, July 18-23, 2010, pp. 3120-3125.

\section{Recommended Citation}

Xiang, Gang and Kreinovich, Vladik, "Towards Improved Trapezoidal Approximation to Intersection (Fusion) of Trapezoidal Fuzzy Numbers: Specific Procedure and General Non-Associativity Theorem" (2010). Departmental Technical Reports (CS). 2.

https://scholarworks.utep.edu/cs_techrep/2

This Article is brought to you for free and open access by the Computer Science at ScholarWorks@UTEP. It has been accepted for inclusion in Departmental Technical Reports (CS) by an authorized administrator of ScholarWorks@UTEP. For more information, please contact Iweber@utep.edu. 


\title{
Towards Improved Trapezoidal Approximation to Intersection (Fusion) of Trapezoidal Fuzzy Numbers: Specific Procedure and General Non-Associativity Theorem
}

\author{
Gang Xiang and Vladik Kreinovich, Senior Member, IEEE
}

\begin{abstract}
In some cases, our uncertainty about a quantity can be described by an interval of its possible values. If we have two or more pieces of interval information about the same quantity, then we can conclude that the actual value belongs to the intersection of these intervals.

In general, we may need a fuzzy number to represent our partial knowledge. A fuzzy number can be viewed as a collection of intervals ( $\alpha$-cuts) corresponding to different degrees $\alpha \in[0,1]$. In practice, we can only store finitely many $\alpha$-cuts. Usually, we only store the lower and upper $\alpha$-cuts (corresponding to $\alpha=0$ and $\alpha=1$ ) and use linear interpolation - i.e., use trapezoidal fuzzy numbers. However, the intersection of two trapezoidal fuzzy numbers is, in general, not trapezoidal. One possible approach is to simply take an intersection of lower and alpha $\alpha$-cuts, but this approach underestimates the resulting membership function.

In this paper, we propose a more accurate approach that uses the Least Squares Method to provide a better linear approximation to the resulting membership function.

While this method provides a more accurate trapezoidal description of the intersection, it has its own drawbacks: e.g., this approximation method makes the corresponding "knowledge fusion" operation non-associative. We prove, however, that this "drawback" is inevitable: specifically, we prove that a perfect solution is not possible, and that any improved trapezoidal approximation to intersection (fusion) of trapezoidal fuzzy numbers leads to non-associativity.
\end{abstract}

\section{INTRODUCTION}

Data fusion: case of interval uncertainty. In some cases, our uncertainty about a quantity can be described by an interval of its possible values; see, e.g., [2].

A typical case of interval uncertainty is when all our information about a quantity comes from measurements. Measuring instruments are never absolutely accurate. As a result, the measured value $\widetilde{x}$ of a quantity is usually somewhat different from the actual (unknown) value $x$ of this quantity. For crude measurements, the difference $\Delta x \stackrel{\text { def }}{=}$ $\widetilde{x}-x$ (or, to be more precise, its absolute value $|\Delta x|$ ) is larger, for accurate measurements this difference $|\Delta x|$ is smaller, but it is always non-zero.

Often, the only information we have about the accuracy of the measuring instrument is the upper bound $\Delta$ on this measurement error: $|\Delta x| \leq \Delta$. In this case, based on the measurement result $\widetilde{x}$, the only information that we can

Gang Xiang is with Philips Healthcare, Business Line RIS, El Paso, TX 79912, USA (phone +1 915 7993621; email: gxiang@sigmaxi.net).

Vladik Kreinovich is with the Department of Computer Science, University of Texas at El Paso, 500 W. University, El Paso, TX 79968, USA (phone +1915 7476951; email: vladik@utep.edu). deduce about the actual (unknown) value $x$ is that $x$ belongs to the interval $X \stackrel{\text { def }}{=}[\widetilde{x}-\Delta, \widetilde{x}+\Delta]$.

Often, we have two or more pieces of interval information $X_{1}, \ldots, X_{n}$ about the same quantity - e.g., coming from two or more different measurements. In this case, we can conclude that the actual value $x$ belongs to the intersection

$$
X=X_{1} \cap \ldots \cap X_{n}
$$

of the corresponding intervals.

The intersection $X=[\underline{x}, \bar{x}]$ is easy to describe in terms of the bounds $X_{i}=\left[\underline{x}_{i}, \bar{x}_{i}\right]$ of the corresponding intervals:

$$
\begin{aligned}
& \underline{x}=\max \left(\underline{x}_{1}, \ldots, \underline{x}_{n}\right) ; \\
& \bar{x}=\min \left(\bar{x}_{1}, \ldots, \bar{x}_{n}\right) .
\end{aligned}
$$

In full accordance with common sense, this operation has the following properties:

- if we combine the same piece of information with itself, the result remains the same $X \cap X=X$; in mathematical terms, this interval "data fusion" operation $\cap$ is idempotent;

- the result of combining several pieces of knowledge does not depend on the order in which we combine them; in particular, this means that

$$
X_{1} \cap X_{2}=X_{2} \cap X_{1},
$$

i.e., in mathematical terms, this "data fusion" operation $\cap$ is commutative, and

$$
X_{1} \cap\left(X_{2} \cap X_{3}\right)=\left(X_{1} \cap X_{2}\right) \cap X_{3},
$$

i.e., in mathematical terms, this interval "data fusion" operation $\cap$ is associative.

Need for fuzzy uncertainty. In many cases, the information about a quantity comes from expert estimates. Experts are often "fuzzy" in their estimates. For example, an expert can say that the value of the quantity is small (without providing us an exact meaning of "small"). In such situations, a natural way to represent this expert information is by using fuzzy numbers; see, e.g., [1], [3].

Data fusion for fuzzy numbers. If we have several fuzzy numbers $X_{1}, \ldots, X_{n}$ describing the same quantity, then it is natural to take their intersection

$$
X=X_{1} \cap \ldots \cap X_{n}
$$


as the fuzzy number that combines (fuses) all these pieces of information.

In terms of membership functions $X_{i}(x)$, the intersection is usually interpreted as the minimum:

$$
X(x)=\min \left(X_{1}(x), \ldots, X_{n}(x)\right) .
$$

Computer representation of fuzzy numbers: trapezoidal numbers. A fuzzy number $X$ can be viewed as a collection of intervals ( $\alpha$-cuts) $X(\alpha)$ corresponding to different degrees $\alpha \in[0,1]: X(\alpha) \stackrel{\text { def }}{=}\{x: X(x) \geq \alpha\}$ for $\alpha>0$ and $X(\alpha) \stackrel{\text { def }}{=} \overline{\{x: X(x)>0\}}$ for $\alpha=0$, where $\bar{S}$ means the closure of the set $S$.

In terms of $\alpha$-cuts, an intersection simply means that for every $\alpha$, we take the intersection of the corresponding $\alpha$-cuts:

$$
X(\alpha)=X_{1}(\alpha) \cap \ldots \cap X_{n}(\alpha) .
$$

In practice, we can only store finitely many $\alpha$-cuts. Usually, we only store the lower and upper $\alpha$-cuts (corresponding to $\alpha=0$ and $\alpha=1$ ) and use linear interpolation - i.e., use trapezoidal fuzzy numbers.

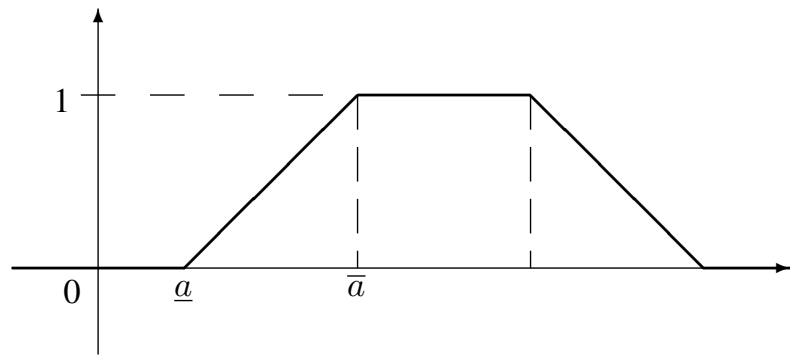

Fusing trapezoidal fuzzy numbers: a problem. The intersection of two trapezoidal fuzzy numbers is, in general, not trapezoidal. It is sufficient to illustrate this fact on the example of the left-hand sides of the intersecting numbers; it is easy to describe a similar example for the right-hand side.

The intersection $\mu_{i}(x)$ of the membership functions

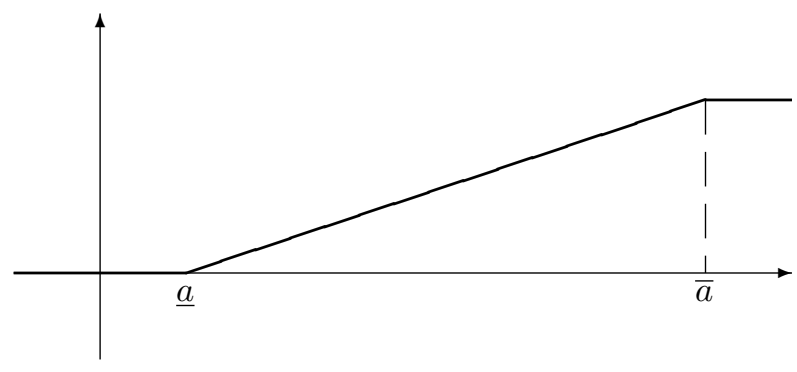

and

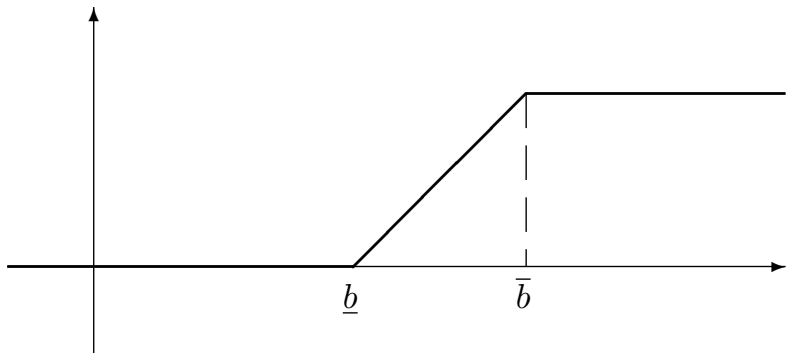

takes the form

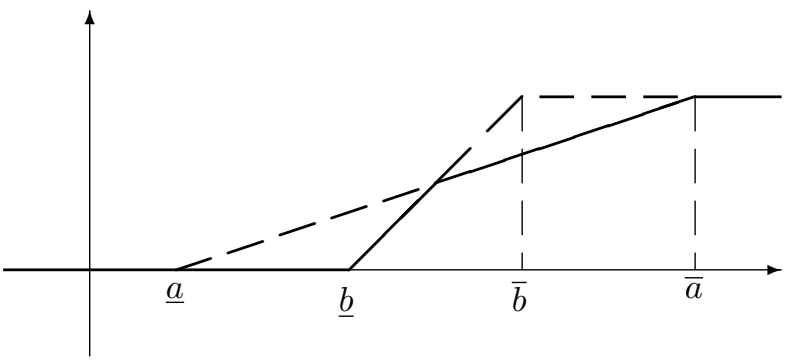

Usual solution. A usual solution to the above problem is simply:

- take an intersection of lower and alpha $\alpha$-cuts, and then

- as the result of the fusion, take the trapezoidal number corresponding to this intersection.

The resulting trapezoidal fuzzy number $\mu_{t}(x)$ is described in the following picture:

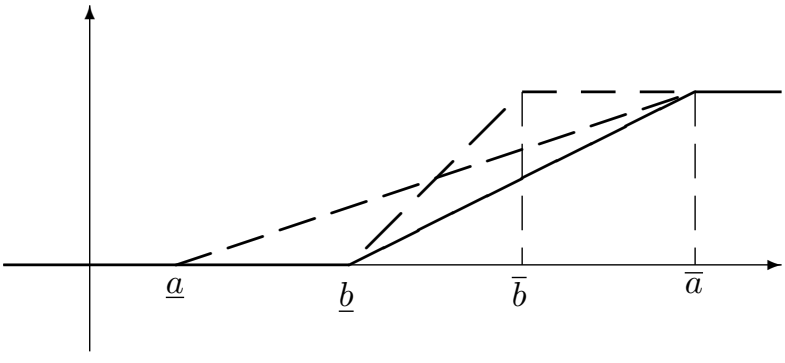

Limitation of the traditional approach. The main problem with this approach is that, as one can easily see, this approach underestimates the resulting membership function.

It is therefore desirable to come up with a different trapezoidal approximation to intersection (fusion) of trapezoidal fuzzy numbers, an approximation that would provide a more accurate approximation to the actual intersection.

Mathematical comment. The above underestimation can be easily explained. Indeed, for both trapezoidal functions, the membership function is linear in the domain where it is different from 0 and 1 . Since we use minimum as the intersection, the combined membership function is a minimum of two linear functions.

A minimum of two linear functions is always concave; see, e.g., [4]. Thus, by definition of a concave function, its values $\mu_{i}(x)$ are always above the straight line $\mu_{t}(x)$ that connects its endpoints, i.e., $\mu_{i}(x) \geq \mu_{t}(x)$ : 


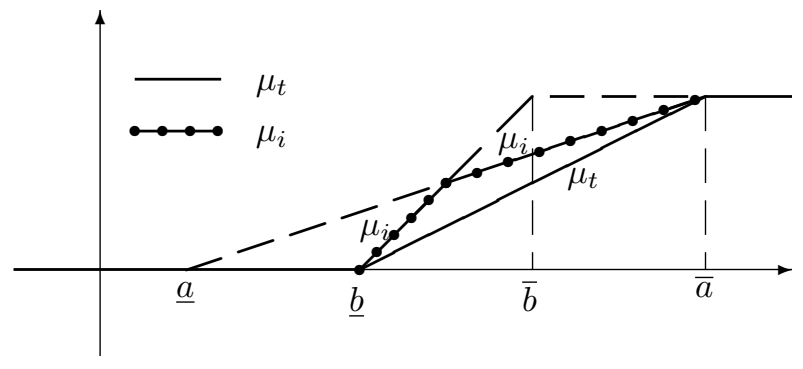

II. How to Improve the TRAditional Trapezoidal Approximation to Intersection: A New Procedure

Analysis of the problem. In the standard approach, we simply take the intersection of the $\alpha$-cuts corresponding to $\alpha=0$ and to $\alpha=1$, and then linearly extrapolate the resulting function. The limitation of this approach is that for intermediate values $\alpha \in(0,1)$, this approach does not provide a good approximation to the $\alpha$-cuts of the intersection.

The resulting idea. The above analysis naturally leads to the following idea:

- First, compute the actual intersection.

- Second, approximate the non-0 and non-1 parts of this intersection by linear functions. For this approximation, we can use, e.g., the usual Least Squares Method; see, e.g., [5].

Least Squares Method: reminder. As we have mentioned, the actual intersection of two trapezoidal membership functions has, in general, two parts where the membership value is different from 0 and 1 . We will approximate each of these parts separately.

Without losing generality, let us consider the interval $[\underline{a}, \bar{a}]$ that contains the left non- 0 non- 1 part. In other words, the membership function $\mu_{i}(x)$ corresponding to the intersection has the property that $\mu_{i}(\underline{a})=0, \mu_{i}(\bar{a})=1$, and

$$
0<\mu_{i}(a)<1
$$

for all values $a$ which are strictly in between $\underline{a}$ and $\bar{a}$. We want to approximate the function $\mu_{i}(a)$ on the interval $[\underline{a}, \bar{a}]$ by a linear function $\mu_{\ell}(a)=p+q \cdot a$ for appropriate values $p$ and $q$. We would like all the values of this linear function to be approximately equal to the values of the original nonlinear membership function:

$$
\mu_{\ell}(a) \approx \mu_{i}(a) \text { for all } a \in[\underline{a}, \bar{a}],
$$

i.e., in other words, that all the approximation inaccuracies

$$
e(a) \stackrel{\text { def }}{=} \mu_{\ell}(a)-\mu_{i}(a)
$$

are approximately zeros:

$$
e(a) \approx 0 \text { for all } a \in[\underline{a}, \bar{a}] .
$$

The traditional Least Squares Method was designed to describe a similar situation when we have finitely many inaccuracies $e_{1}, \ldots, e_{n}$ all of which needs to be approximately equal to 0 . This situation can be alternatively described by saying that the point $e \stackrel{\text { def }}{=}\left(e_{1}, \ldots, e_{n}\right)$ in an $n$-dimensional space should be approximately equal to the zero point $0=(0, \ldots, 0)$. Different approximations are characterized by different points $e$. A reasonable idea is to select an approximation for which the resulting point $e$ is the closest to the 0 point, i.e., for which the distance $\rho(e, 0)$ between these two points is the smallest possible. In this formulation, it is natural to use the Euclidean metric

$$
\rho(a, b)=\sqrt{\left(a_{1}-b_{1}\right)^{2}+\ldots+\left(a_{n}-b_{n}\right)^{2}} .
$$

For this metric,

$$
\rho(e, 0)=\sqrt{e_{1}^{2}+\ldots+e_{n}^{2}} .
$$

Instead of minimizing this distance, it is usually more computationally convenient to solve an equivalent problem of minimizing the square of this distance. Indeed, when the approximating expression linearly depends on the unknown parameters - as in our case, when $e(a)=p+q \cdot a-\mu_{\ell}(a)$ is a linear function of $p$ and $q$ - the sum of the squares is a quadratic functions of the unknown parameters. Thus, when we optimize the objective function by differentiating it with respect to each of these parameters and equating the derivatives to 0 , we get an (easy-to-solve) system of linear equations for determining the desired values of the parameters. For the square root expressions, derivatives are more complicated.

Because of this computations-related trick, the Least Squares method means finding the values of the parameters for which the sum of the squares of inaccuracies attains the smallest possible ("least") value:

$$
\text { Minimize } e_{1}^{2}+\ldots+e_{n}^{2} \text {. }
$$

Least Squares Method: continuous case. In our case, instead of finitely many inaccuracy values $e_{1}, \ldots, e_{n}$, we have inaccuracy values $e(a)$ corresponding to all (infinitely many) values $a$ from the interval $[\underline{a}, \bar{a}]$. This case can be considered as a limit of cases in which we have a larger and larger number of points $a_{i}$ which are more and more densely located on the interval $[\underline{a}, \bar{a}]$. In this limit, the above sum turns into the corresponding integral

$$
\int_{\underline{a}}^{\bar{a}}\left(\mu_{\ell}(a)-\mu_{i}(a)\right)^{2} d a,
$$

i.e., into

$$
\int_{\underline{a}}^{\bar{a}}\left(p+q \cdot a-\mu_{i}(a)\right)^{2} d a .
$$

Least Squares Method: towards the formulas. Differentiating the above expression with respect to $p$ and $q$ and equating the derivatives to 0 , we conclude that

$$
\int_{\underline{a}}^{\bar{a}}\left(p+q \cdot a-\mu_{i}(a)\right) d a=0
$$




$$
\int_{\underline{a}}^{\bar{a}}\left(p+q \cdot a-\mu_{i}(a)\right) \cdot a d a=0 .
$$

Taking into account that the integral of the sum is equal to the sum of the integrals, explicitly integrating the terms proportional to $p$ and $q$, and moving all other terms to the right-hand side, we get the following system of two linear equations for determining the unknowns $p$ and $q$ :

$$
\begin{gathered}
p \cdot(\bar{a}-\underline{a})+q \cdot \frac{(\bar{a})^{2}-(\underline{a})^{2}}{2}=\int_{\underline{a}}^{\bar{a}} \mu_{i}(a) d a ; \\
p \cdot \frac{(\bar{a})^{2}-(\underline{a})^{2}}{2}+q \cdot \frac{(\bar{a})^{3}-(\underline{a})^{3}}{3}=\int_{\underline{a}}^{\bar{a}} \mu_{i}(a) \cdot a d a .
\end{gathered}
$$

The intersection membership function $\mu_{i}(a)$ is piece-wise linear, consisting of two linear parts. Thus, the corresponding integrals can be explicitly computed by considering both linear parts and adding the results. We can then use the explicit formulas for solving a system of two linear equations to get an explicit expression for $p$ and $q-$ and thus, for $\mu_{\ell}(a)=p+q \cdot a$. This linear membership function $\mu_{\ell}(a)$ is then used as the desired approximation to $\mu_{i}(a)$ :

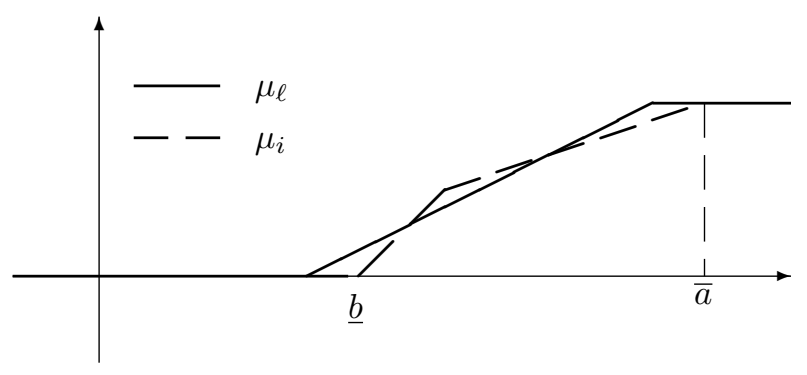

\section{Problem With the NeW Approximation PROCEDURE: NON-ASSOCIATIVITY}

A somewhat unexpected problem: non-associativity. We tried the above Least Squares approach to combine two trapezoidal membership functions $X_{1}$ and $X_{2}$ into a single trapezoidal membership function $X_{1} \otimes X_{2}$ that approximates the intersection $X_{1} \cap X_{2}$. We saw that there is an unexpected problem with this approach: while the resulting operation is still commutative, this operation is no longer associative.

Specifically, when we take three different pieces of knowledge $X_{1}, X_{2}$, and $X_{3}$, then we can combine them in several different ways:

- we can first combine $X_{1}$ and $X_{2}$ into a trapezoidal function $X_{1} \otimes X_{2}$, and then combine the result $X_{1} \otimes X_{2}$ with $X_{3}$ into a new membership function

$$
\left(X_{1} \otimes X_{2}\right) \otimes X_{3}
$$

- alternatively, we can first combine $X_{2}$ and $X_{3}$ into a trapezoidal function $X_{2} \otimes X_{3}$, and then combine $X_{1}$ with the result $X_{2} \otimes X_{3}$ into a new membership function

$$
X_{1} \otimes\left(X_{2} \otimes X_{3}\right) \text {. }
$$

In both ways, we combine all three pieces of knowledge. So, intuitively, we expect the results to be equal

$$
\left(X_{1} \otimes X_{2}\right) \otimes X_{3}=X_{1} \otimes\left(X_{2} \otimes X_{3}\right) .
$$

In mathematical terms, we expect the combination operation $\otimes$ to be associative.

In practice, however, the Least Squares-based operation $\otimes$ is not associative.

Comment. A numerical example is presented later in the text, in the proof of our main result.

Non-associativity is indeed a problem. One may argue that the above non-associativity is not always a problem. For example, if from the very beginning, we have all three pieces of knowledge $X_{1}, X_{2}$, and $X_{3}$, then we can simply take the intersection $X_{1} \cap X_{2} \cap X_{3}$ of all three of them, and then use the Least Squares (or any other approximation technique) to find the trapezoidal function that approximates this intersection.

In many practical situations, however, pieces of knowledge are not available from the very beginning, they arrive one by one. If we first have $X_{1}$, after which $X_{2}$ arrives, then we approximate the resulting knowledge by a trapezoidal membership function $X_{1} \otimes X_{2}$. If after this, a new piece of knowledge $X_{3}$ arrives, then combine this piece of knowledge with the current state $X_{1} \otimes X_{2}$ of our knowledge base, resulting in $\left(X_{1} \otimes X_{2}\right) \otimes X_{3}$.

Alternatively, if we first have $X_{2}$, after which $X_{3}$ arrives, then we approximate the resulting knowledge by a trapezoidal membership function $X_{2} \otimes X_{3}$. If after this, a new piece of knowledge $X_{1}$ arrives, then combine this piece of knowledge with the current state $X_{2} \otimes X_{3}$ of our knowledge base, resulting in $X_{1} \otimes\left(X_{2} \otimes X_{3}\right)$.

In both cases, eventually, we learn the same three pieces of knowledge $X_{1}, X_{2}$, and $X_{3}$. It is therefore desirable in both case, we should end up in the same state of our knowledgebase, i.e., that we should have

$$
\left(X_{1} \otimes X_{2}\right) \otimes X_{3}=X_{1} \otimes\left(X_{2} \otimes X_{3}\right) .
$$

Resulting question. Associativity seems to be desirable. The Least Squares method does not lead to associativity. Since there are many other approximation techniques beyond the Least Squares, a natural question is: can we retain associativity by using one of these alternative techniques?

Non-associativity is inevitable. In the remaining part of this paper, we prove that non-associativity is inevitable: no matter what approximation procedure we select, the resulting approximation operation is not associative.

Thus, the only way to avoid non-associativity is to use the traditional (underestimating) trapezoidal description of the intersection of two trapezoidal membership functions.

\section{Properties of the General Approximation OPERATION: MOTIVATIONS}

Plan of work. We would like to prove that every "reasonable" approximation operation $\otimes$ is not associative. To prove this result, we will first describe properties that are reasonable to require of any operation that approximates an intersection 
with a trapezoid. These properties will be described in this section.

Then, in the next section, we will prove that every operation $\otimes$ that satisfies these reasonable properties is not associative.

First idea: we only consider left sides. As we have mentioned, it is sufficient to separately consider the left and the right sides of the corresponding membership functions. Since the right sides are similar to the left ones, we will only analyze the left sides.

In any case, this restriction to left sides is sufficient for our purpose: if the combination of left sides is not associative, then the whole combination operation is not associative either.

From the mathematical viewpoint, the left side of a trapezoidal membership is an interval. To describe the left side of a trapezoidal membership function $\mu$, it is sufficient to describe two values:

- the last value $\underline{a}$ for which $\mu(\underline{a})=0$ and

- the first value $\bar{a}$ for which $\mu(\bar{a})=1$ :

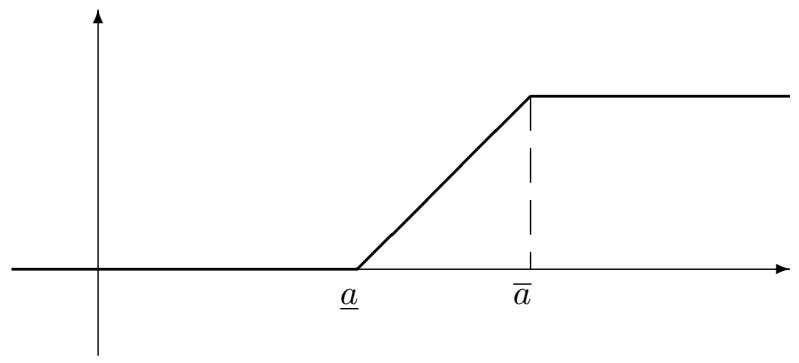

The value $\underline{a}$ is always smaller than or equal to the value $\bar{a}$. Thus, these values form an interval $\mathbf{a}=[\underline{a}, \bar{a}]$. Vice versa, once we have an interval, $\mathbf{a}=[a, \bar{a}]$, we can form a (left side of the) trapezoidal function by selecting a function $\mu(x)$ which:

- is equal to 0 for $x \leq \underline{a}$;

- is equal to 1 for $x \geq \bar{a}$, and

- is linear on the interval $[\underline{a}, \bar{a}]$, i.e., is equal to $\mu(x)=$ $\frac{x-\underline{a}}{\bar{a}-\underline{a}}$ for $\underline{a}<x<\bar{a}$.

Since left sides of trapezoidal membership functions are thus in natural 1-1 correspondence with intervals, we will whenever it is more convenient - talk about the operation $\otimes$ on intervals.

First property: commutativity. The result of combining two pieces of knowledge should not depend on the order in which these two pieces of knowledge are listed, i.e., we should have $\mathbf{a} \otimes \mathbf{b}=\mathbf{b} \otimes \mathbf{a}$ for all intervals $\mathbf{a}$ and $\mathbf{b}$.

Second property: idempotence $\mathbf{a} \otimes \mathbf{a}=\mathbf{a}$. If the new piece of knowledge $X_{2}$ is identical to our original knowledge $X_{1}$ $\left(X_{1}=X_{2}\right)$, this means that this new piece of knowledge does not provide us with any new information. Thus, we do not gain anything new by combining these two pieces of knowledge $X_{1}$ and $X_{2}$.
In other words, the result $X_{1} \otimes X_{1}$ of combining every piece of knowledge with itself should be identical to that original piece of knowledge: $X_{1} \otimes X_{1}=X_{1}$. This means that for every interval $\mathbf{a}$, we must have $\mathbf{a} \otimes \mathbf{a}=\mathbf{a}$, i.e., in mathematical terms, that the interval operation $\otimes$ is idempotent.

Third property: preserving trapezoidal intersections. A slightly more general case where we are sure what we want as a result $\mathbf{a} \otimes \mathbf{b}$ is when the intersection $\mathbf{a} \cap \mathbf{b}$ of the corresponding membership functions is itself trapezoidal. In this case, the trapezoidal approximation to this intersection membership function should, of course, coincide with this intersection.

One can easily see that the case when the intersection of the left sides corresponding to the intervals $\mathbf{a}=[\underline{a}, \bar{a}]$ and $\mathbf{b}=[\underline{b}, \bar{b}]$ is itself trapezoidal is when this intersection coincides with one of the original membership functions, i.e., when we have one of the following two cases.

In the first case, we have $\underline{a} \leq \underline{b}$ and $\bar{a} \leq \bar{b}$. In this case, the intersection is equal to the membership function described by the interval $\mathbf{b}$ :

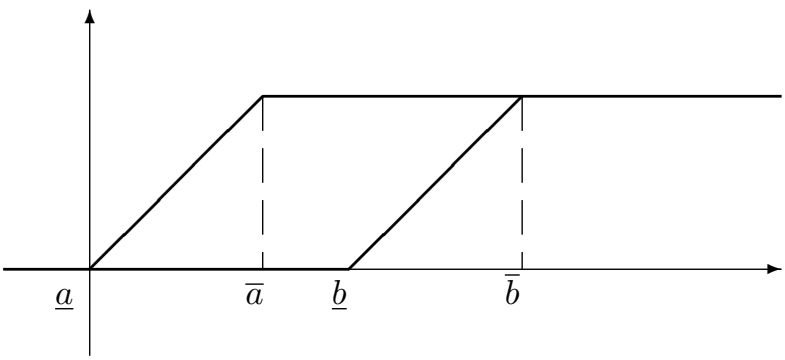

In the second case, we have $\underline{b} \leq \underline{a}$ and $\bar{b} \leq \bar{a}$. In this case, the intersection is equal to the membership function described by the interval a.

For convenience, we will denote the relation " $\underline{a} \leq \underline{b}$ and $\bar{a} \leq \bar{b}$ " between the two intervals $\mathbf{a}=[\underline{a}, \bar{a}]$ and $\mathbf{b}=[\underline{b}, \bar{b}]$ as $a \leq b$. In terms of this notation, the above requirement is that if $\mathbf{a} \leq \mathbf{b}$, then $\mathbf{a} \otimes \mathbf{b}=\mathbf{b}$.

Fourth property: the combination operation should be an improved approximation. For the non-trivial case, when $\mathbf{a} \not \leq \mathbf{b}$ and $\mathbf{b} \not \leq \mathbf{a}$, we would like to require that the upper endpoint of the resulting interval $\mathbf{c}=\mathbf{a} \otimes \mathbf{b}$ must be strictly in between the upper endpoints of the combined intervals, and the lower endpoint of $\mathbf{c}$ must also be strictly in between the corresponding endpoints:

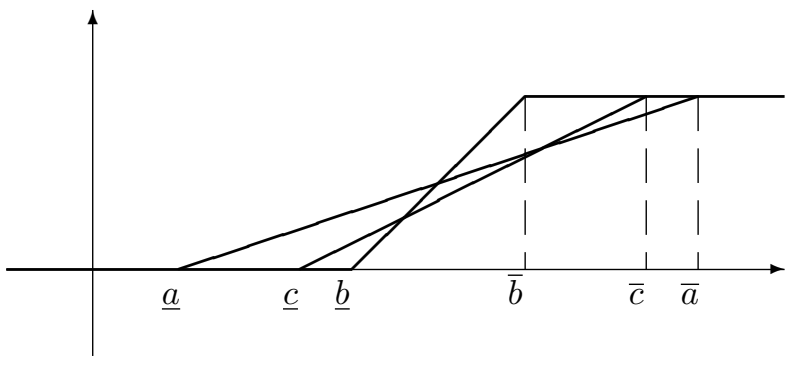


Thus, we would like to require that in this case, $\mathbf{a} \not \leq \mathbf{a} \otimes \mathbf{b}$, $\mathbf{a} \otimes \mathbf{b} \not \leq \mathbf{a}, \mathbf{b} \not \leq \mathbf{a} \otimes \mathbf{b}$, and $\mathbf{a} \otimes \mathbf{b} \not \leq \mathbf{b}$.

Now, we are ready to formulate our main result.

\section{DeFinitions AND THE General NON-ASSOCIATIVITY THEOREM}

Notation. For two intervals $\mathbf{a}=[\underline{a}, \bar{a}]$ and $\mathbf{b}=[\underline{b}, \bar{b}]$, we say that $\mathbf{a} \leq \mathbf{b}$ if $\underline{a} \leq \underline{b}$ and $\bar{a} \leq \bar{b}$.

Definition. By an improved trapezoidal approximation to the intersection of trapezoidal fuzzy numbers, we mean an operation $\otimes$ that transforms any two intervals $\mathbf{a}$ and $\mathbf{b}$ into an interval $\mathbf{a} \otimes \mathbf{b}$, and which satisfies the following properties:

- it is commutative, i.e., $\mathbf{a} \otimes \mathbf{b}=\mathbf{b} \otimes \mathbf{a}$ for all intervals $\mathbf{a}$ and $\mathbf{b}$;

- it is idempotent, i.e., a $\otimes \mathbf{a}$ for all intervals $\mathbf{a}$;

- it preserves trapezoidal intersection, i.e., if $\mathbf{a} \leq \mathbf{b}$, then $\mathbf{a} \otimes \mathbf{b}=\mathbf{b}$

- it improves over the usual approximation, i.e., if $\mathbf{a} \not \leq \mathbf{b}$ and $\mathbf{b} \not \leq \mathbf{a}$, then $\mathbf{a} \not \leq \mathbf{a} \otimes \mathbf{b}, \mathbf{a} \otimes \mathbf{b} \not \leq \mathbf{a}, \mathbf{b} \not \leq \mathbf{a} \otimes \mathbf{b}$, and $\mathbf{a} \otimes \mathbf{b} \not \mathbf{b}$.

Proposition. Every improved trapezoidal approximation to the intersection of trapezoidal fuzzy numbers is nonassociative.

Comment. In other words, for every such operation $\otimes$, there exist trapezoidal membership functions $X_{1}, X_{2}$, and $X_{3}$ for which

$$
\left(X_{1} \otimes X_{2}\right) \otimes X_{3} \neq X_{1} \otimes\left(X_{2} \otimes X_{3}\right) .
$$

In terms of intervals, for every operation $\otimes$, there exist intervals $\mathbf{a}, \mathbf{b}$, and $\mathbf{c}$, for which

$$
(\mathbf{a} \otimes \mathbf{b}) \otimes \mathbf{c} \neq \mathbf{a} \otimes(\mathbf{b} \otimes \mathbf{c}) .
$$

Proof. Let us pick two intervals $\mathbf{a}$ and $\mathbf{b}$ for which $\mathbf{a} \not \leq \mathbf{b}$ and $\mathbf{b} \not \leq \mathbf{a}$. For example, we can take $\mathbf{a}=[1,4]$ and $\mathbf{b}=[2,3]$. Let us show that associativity is violated for $\mathbf{c}=\mathbf{b}$. We will prove this by reduction to a contradiction. Let us assume that associativity is satisfied for these three intervals, i.e., that

$$
(\mathbf{a} \otimes \mathbf{b}) \otimes \mathbf{b}=\mathbf{a} \otimes(\mathbf{b} \otimes \mathbf{b}) .
$$

Due to idempotence, we have $\mathbf{b} \otimes \mathbf{b}=\mathbf{b}$, so the above equality leads to

$$
(\mathbf{a} \otimes \mathbf{b}) \otimes \mathbf{b}=\mathbf{a} \otimes \mathbf{b} .
$$

So, for $\mathbf{d} \stackrel{\text { def }}{=} \mathbf{a} \otimes \mathbf{b}$, we have $\mathbf{d} \otimes \mathbf{b}=\mathbf{d}$ and thus, $\mathbf{d} \leq \mathbf{d} \otimes \mathbf{b}$. One of our requirements is that the operation $\otimes$ is improving, meaning that $\mathbf{b} \not \leq \mathbf{d}$ and $\mathbf{d} \not \leq \mathbf{b}$ would imply that $\mathbf{d} \not \leq \mathbf{d} \otimes \mathbf{b}$. Thus, the fact that $\mathbf{d} \leq \mathbf{d} \otimes \mathbf{b}$ means that we have either $\mathbf{b} \leq \mathbf{d}$ or $\mathbf{d} \leq \mathbf{b}$.

However, since we assumed that $\mathbf{a} \not \leq \mathbf{b}$ and $\mathbf{b} \not \leq \mathbf{a}$, we can use the same improvement property to conclude that $\mathbf{b} \not \subset$ $\mathbf{d}=\mathbf{a} \otimes \mathbf{b}$ and $\mathbf{d}=\mathbf{a} \otimes \mathbf{b} \not \leq \mathbf{b}$. The contradiction proves that the above equality cannot be true. So, associativity is indeed violated. The proposition is proven.

\section{ACKNOWLEDGMENTS}

This work was supported in part by the National Science Foundation grants HRD-0734825 and DUE-0926721, by Grant 1 T36 GM078000-01 from the National Institutes of Health, by Grant MSM 6198898701 from MŠMT of Czech Republic, and by Grant 5015 "Application of fuzzy logic with operators in the knowledge based systems" from the Science and Technology Centre in Ukraine (STCU), funded by European Union.

The authors are greatly thankful to Ronal R. Yager for valuable discussions, and to the anonymous referees for useful advise.

\section{REFERENCES}

[1] G. Klir and B. Yuan, Fuzzy Sets and Fuzzy Logic: Theory and Applications, Upper Saddle River, New Jersey: Prentice Hall, 1995.

[2] R. E. Moore, R. B. Kearfott, and M. J. Cloud, Introduction to Interval Analysis, Philadelphia, Pennsylvania: SIAM Press, 2009.

[3] H. T. Nguyen and E. A. Walker, A First Course in Fuzzy Logic, Boca Raton, Florida: Chapman \& Hall/CRC, 2006.

[4] R. T. Rockafellar, Convex Analysis, Princeton University Press, Princeton, 1996.

[5] J. Wolberg, Data Analysis Using the Method of Least Squares: Extracting the Most Information from Experiments, Springer Verlag, Berlin Heidelberg New York, 2005. 